\title{
Bringing consistency to simulation of population models - Poisson Simulation as a bridge between micro and macro simulation
}

\author{
Leif Gustafsson $^{\mathrm{a}, *}$, Mikael Sternad ${ }^{\mathrm{b}}$ \\ ${ }^{a}$ Department of Biometry and Engineering, Swedish University of Agricultural Sciences, \\ P.O. Box 7032, SE-750 07 Uppsala, Sweden \\ ${ }^{\mathrm{b}}$ Signals and Systems, Department of Engineering and Sciences, Uppsala University, Uppsala, Sweden
}

Received 18 September 2006; received in revised form 25 January 2007; accepted 19 February 2007

Available online 28 February 2007

\begin{abstract}
Population models concern collections of discrete entities such as atoms, cells, humans, animals, etc., where the focus is on the number of entities in a population. Because of the complexity of such models, simulation is usually needed to reproduce their complete dynamic and stochastic behaviour. Two main types of simulation models are used for different purposes, namely micro-simulation models, where each individual is described with its particular attributes and behaviour, and macro-simulation models based on stochastic differential equations, where the population is described in aggregated terms by the number of individuals in different states. Consistency between micro- and macro-models is a crucial but often neglected aspect. This paper demonstrates how the Poisson Simulation technique can be used to produce a population macro-model consistent with the corresponding micro-model. This is accomplished by defining Poisson Simulation in strictly mathematical terms as a series of Poisson processes that generate sequences of Poisson distributions with dynamically varying parameters. The method can be applied to any population model. It provides the unique stochastic and dynamic macro-model consistent with a correct micro-model. The paper also presents a general macro form for stochastic and dynamic population models. In an appendix Poisson Simulation is compared with Markov Simulation showing a number of advantages. Especially aggregation into state variables and aggregation of many events per time-step makes
\end{abstract}

\footnotetext{
${ }^{*}$ Corresponding author. Tel.: +46 18 500061; fax: +46 18673529.

E-mail address: Leif.Gustafsson@bt.slu.se (L. Gustafsson).
} 
Poisson Simulation orders of magnitude faster than Markov Simulation. Furthermore, you can build and execute much larger and more complicated models with Poisson Simulation than is possible with the Markov approach.

(c) 2007 Elsevier Inc. All rights reserved.

Keywords: Consistency; Population model; Poisson process; Poisson Simulation; Stochastic SIR model; Stochastic difference equation

\section{Introduction}

Population models [1-3] concern collections of discrete entities such as atoms, molecules, genes, cells, humans, animals, plants, etc. and are frequently studied in physics, biology, ecology, demography, epidemiology, production, queuing systems and many other fields where processes with discrete entities develop over time. In population models, the integer number of individuals/entities in a population or sub-population is the main property of interest.

Since a studied system, being part of the real world, is immensely complex, it is crucial to construct a conceptual model where the components, structure and logics are comprehensible. This model represents our basic knowledge and assumptions as we understand the system. Although we realise that the conceptual model is not the full truth of the system, the task is to reformulate it into a form that can be examined without further distorting the model in the reformulation.

For a few very simple models, mathematics and statistics can be used to calculate the dynamics and/or stochastic variations. The most powerful tool for theoretical analysis of population models is probably Markov theory [4,5]. However, a number of more or less distorting simplifications, modifications and additional assumptions are usually needed to perform a mathematical or statistical analysis even on rather simple models. Simulation is then a powerful tool to describe the dynamic and stochastic behaviour of the conceptual model. Discrete Markov models are also frequently used for simulation of population models, but this approach has a number of disadvantages compared with Poisson Simulation. The huge set of states already for moderate size Markov models is replaced by a small number of state variables in Poisson Simulation modelling. This aggregation into state variables and aggregation of many events per time-step makes Poisson Simulation orders of magnitude faster than Markov Simulation. Furthermore, you can build and execute much larger and more complicated models with Poisson Simulation than is possible with the Markov approach. This is discussed in more detail in Appendix C.

When reformulating the conceptual population model into an operative simulation model, one can choose between a micro-model approach describing every individual and a macro-model approach aggregating the individuals into classes/states of individuals where only the number of individuals in each state is described. These types of models are needed for different perspectives.

Micro-models focus on the individual, with his/her/its unique attributes and behaviours and can therefore handle both homogeneous and heterogeneous populations. The translation of the conceptual model into a numerical setting is usually technically simple and straight-forward, and can be performed in Discrete Event Simulation (DES) [6,7] or Individual Based Simulation [8]. This 
approach is based on computer programming where operations on individuals are stochastic. Since this approach has to deal with each individual interacting alone or with other individuals, the computational effort increases strongly with the size of the population. Although modern computers can handle extremely large models, this is still a formidable task for large populations, requiring many replications when model fitting, optimisation or parameter estimation is involved. The micro-models play almost no role in the theoretical analysis.

Macro-models are used for the large perspective. These models can be based on deterministic or stochastic differential equations, where all individuals of a state are aggregated into one variable. Therefore, the computational effort becomes independent of the size of the population studied. Here, the possible heterogeneity is limited by the state aggregation, where only the numbers of entities in different states are modelled as in Continuous System Simulation (CSS) $[9,10]$. Macro-models are also more or less approachable by mathematical and statistical methods and are therefore also important for theoretical results. However, translation of the population dynamics and stochastics into differential/difference equation form is not trivial. In particular, omitting the stochasticity or the discrete nature of the individuals often leads to absurd results.

The duality of the micro- and macro-model approaches is important because micro-models are very flexible when the population under investigation is heterogeneous and different attributes (e.g., age, sex, variety, location, etc.) of different individuals need to be taken into account, while macro-models are excellent at treating the large-scale perspective and are more accessible for theoretical analysis. It is also important that results such as outcomes, statistical estimates, risks, estimated parameter values, etc. are consistent (contradiction-free), regardless of whether the approach used is micro or macro. Results from different studies can then be compared and results such as estimated values of parameters can be transferred between consistent micro- and macromodels. Such consistency between micro- and macro-models is thus a very important but often neglected aspect of population analysis.

This paper demonstrates how the Poisson Simulation [11] technique (preserving the stochastics and individual nature of the entities) can be used for macro-modelling to produce a population model consistent with that when a micro approach is used. A strict mathematical description of the Poisson Simulation method is presented for the first time. This enables us to formulate a mathematically correct macro-simulation representation of a population model and provides a tool to prove the consistency between micro and macro approaches of such a model. In the end of this paper, a general form for stochastic and dynamic population models is also presented.

Poisson Simulation is a stochastic extension of Continuous System Simulation. It is based on a form of differential/difference equation that models change as purely stochastic rather than as the sum of a deterministic and a stochastic part. For example, radioactive decay is modelled by

$$
\mathrm{d} x(t)=P o[\mathrm{~d} t \cdot a x(t)],
$$

(where $P o[\cdot]$ means a Poisson distributed deviate of the argument in the brackets) rather than by $\mathrm{d} x(t) / \mathrm{d} t=a x(t)+e(t)$. The latter equation implies a number of unfeasible phenomena, inconsistent with our physical knowledge, such as non-integer numbers of atoms, stochastic variations unrelated to the remaining number of atoms, sudden increases in the number of atoms, continued variations around steady state (zero), which here means a negative number of atoms, etc. Such artefacts are eliminated by the Poisson approach. Furthermore, no white noise (with its infinite 
energy content) is involved in the Poisson approach. Another advantage with the Poisson approach in its numerical form is that the step-size $\Delta t$ is freely scalable without affecting parameter values, just as deterministic models are in Continuous System Simulation.

The main objective of this paper is to present a method to realise a given, well-specified, conceptual population model as a correct micro- or a macro-simulation model preserving the consistency, in terms of introducing no contradictions, between these models. A proof for the consistency is also given. The scope is restricted to stochastic population models in continuous time. (In Appendix B the corresponding discrete-time modelling is discussed.)

For a given conceptual model, the description of individuals and the implementation of the logics and stochastics into a micro-model are usually straight-forward since every individual can be fully controlled. The full agreement with the conceptual model then follows from the construction of the micro-model. The main emphasis of this paper, therefore, is on how a correct macro-model can be constructed from the conceptual model by using the differential equation description of Continuous System Simulation extended by the Poisson Simulation approach.

A conceptual model together with its micro- and macro-realisations are shown in Fig. 1, which also gives an overview of the analytical process carried out.

Thus, we first define a conceptual model. From this model we construct a micro-model where each individual exactly follows the logic of the conceptual model. In addition, we construct an aggregated macro-model where all individuals in each state are represented by a number. Then we prove the consistency between the micro- and macro-models, which is the main result of the analysis, since if both models are correct realisations of the conceptual model, they should also produce consistent results. For reasons of clarity, we illustrate this by carrying out 10000 simulations of the stochastic micro- and macro-models to compare their results. We also compare

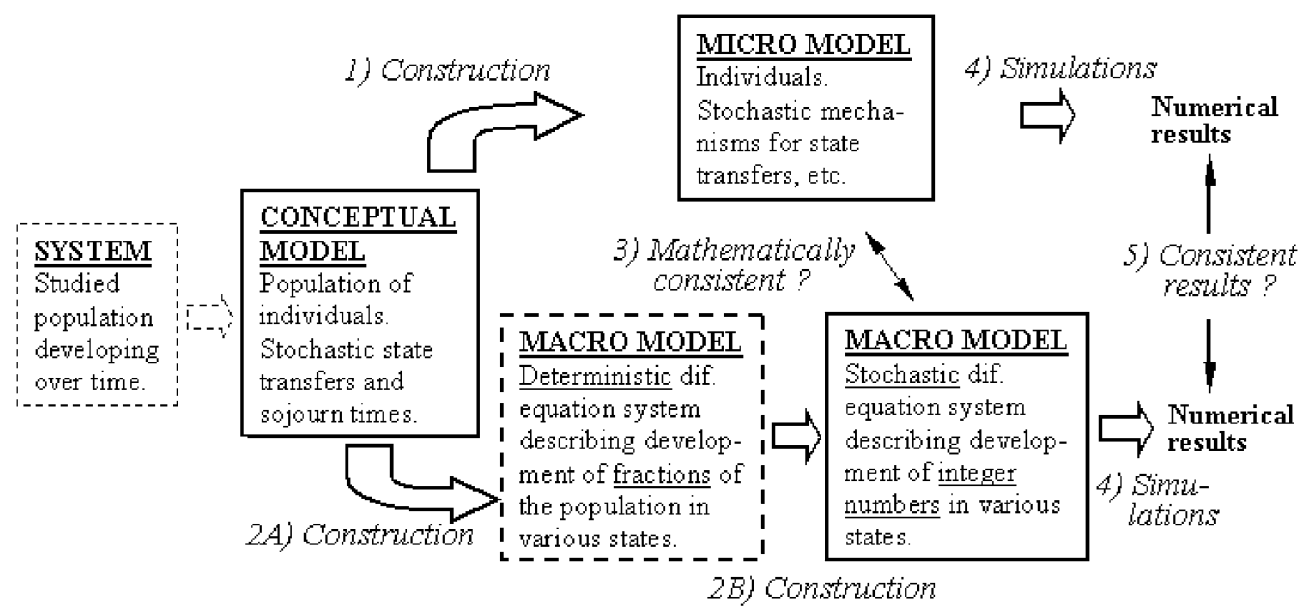

Fig. 1. Overview of the analytical process. Starting from a conceptual model of a studied system, (1) a micro-simulation model based on individuals is constructed and (2A) a deterministic macro-simulation model based on differential equations of fractions of the population is developed. In a second step (2B), the macro-model is reconstructed to be stochastic and based on integer numbers of individuals. In step (3), the mathematical consistency between the microand macro-models is proved. Then (4) the micro- and macro-models are simulated and (5) the results are compared. 
our results with those of a deterministic model embedded in the stochastic macro-model, to show what can happen when discreteness and stochasticity are absent.

\subsection{Events and activities}

Population models include a dynamic and stochastic description of a process. Examples of this are models of radioactive decay of atoms, chemical reactions between molecules, demographic development, queuing systems of humans or parts in an assembly line, biological growth in numbers, biological competition or predation, epidemic processes, cancer development on cell level, Lanchester combats and many others where discrete entities act in continuous time. The dynamic and stochastic processes are based on one or both of two fundamental mechanisms which we denote event and activity (or duration) using concepts from Discrete Event Simulation [12].

An event is something that is modelled as happening instantly, with no duration. For example the decay of an atom, the arrival of a customer, the breakdown of a machine or the infection of an individual.

An activity is a process with a duration such as the time in a queue, the travel time or the duration of a disease. It can be described by a start and an end event referring to the same individual. The necessity to separate activity from events (instead of reducing it to two events) is clear in Discrete Event Simulation, where activities with the same average duration can have different statistical distributions such as uniform, exponential, Weibull, etc. In Continuous System Simulation there is no way to refer to a specific individual in a state. In the simple case where the individuals in a state have the same probability of departing (regardless of how long they have been in the state) we have implicitly an exponential distribution for the residence time in that state. In this type of simulation, other statistical distributions must be achieved or approximated by structures of states in series and/or parallel [13].

\subsection{Selection of a population model}

The specific objective of the paper is to demonstrate how any well-defined model (realistic or not) can be modelled in micro and in macro terms without losing consistency. For this purpose we require a model with the following qualities:

- The model must include the two mechanisms: Event and Activity.

- We prefer a non-linear model.

- The model should otherwise be as simple as possible.

- For pedagogic reasons it is an advantage to use a well-known model.

To make the presentation more concrete, an epidemic SIR model (presented below) is chosen as a representative example of stochastic population models. The choice of a simple SIR model is thus, only to demonstrate the ideas that are general for population models and there is no intention whatsoever to deal with different aspects of SIR models. We could equally well have used, e.g., a logistic model where reproduction is delayed or a Volterra model with an unfertile juvenile stage or a Lanchester combat model with a delay for reloading weapons, etc. 


\section{A conceptual model}

An epidemic is a process that affects a population of individuals, where each individual is an entity with attributes such as stage of disease, sex, age, occupation and behavioural patterns dictating where individuals go, who they meet, how they act when ill, etc.

Models of infectious diseases are generally based on a sequence of stages from Susceptible via Infectious to Recovered/Removed. Such a model is therefore denoted a SIR model. The first SIR model was published by Kermack and McKendrick in 1927 [14] and since then books on the epidemiology of infectious diseases are usually based on the SIR and related models [1,15-17].

For the purposes of the present work, a conceptual model is defined, where the fundamental structure and assumptions about the population and the disease are stated. The crucial point is to obtain a well-defined standard when later constructing the micro- and macro-simulation models, while the realism for a specific disease is outside the scope of this work. Lack of perfect information about who meets whom, whether the Susceptible individual becomes infected, sojourn times in Infectious stages, etc. makes it necessary to use statistical concepts such as risk, statistical distribution, independency, etc.

The conceptual model considered here has the following setting: A population consisting of individuals is affected by an infectious disease. The population is homogeneous, i.e., there are no differences between individuals due to age, sex, susceptibility, behaviour, etc.

The individuals are in one of three consecutive stages $(\mathbf{S}, \mathbf{I}$ or $\mathbf{R})$. Transfer from $\mathbf{S}$ to $\mathbf{I}$ can take place because every individual can meet every other under equal conditions at each time unit with the same probability, $m$, and when a Susceptible individual meets an Infectious individual there is a small risk, $r$, to the former of being infected. To make the conceptual model as simple as possible we use a single parameter, $p=m \cdot r$, for the combined probability of a Susceptible individual meeting an Infectious individual per time unit and thereby becoming infected. The transfer from $\mathbf{I}$ to $\mathbf{R}$ takes place when an Infectious individual has been in stage I for a certain time. This expected time that an individual resides in stage $\mathbf{I}$ (an activity) has an exponential distribution with the mean of $T$ time units.

The change of stage for an individual is called an event and takes place instantaneously. Furthermore we assume that an event is statistically independent of all other events at that point in time.

In a condensed mathematical form, used by, e.g., Bartlett [1], the infinitesimal transition probabilities can be expressed as:

$$
P[(S, I, R) \rightarrow(S-1, I+1, R)]=p \cdot I \cdot S \cdot \mathrm{d} t \text { and } P[(S, I, R) \rightarrow(S, I-1, R+1)]=I \cdot \mathrm{d} t / T .
$$

Comment. A numerical approximation of $\mathrm{d} S=-S \cdot I \cdot p \cdot \mathrm{d} t$ with $\Delta S=-S \cdot I \cdot p \cdot \Delta t$ is often used in macro modelling. This is a good numerical approximation for small time-steps, $\Delta t$, but it is not logically exact for a finite $\Delta t$.

In the conceptual model, it is assumed that every Susceptible individual can meet every Infectious individual during a time unit with the probability $m$ and with the risk $r$ of getting infected in a meeting. Then, for example, a Susceptible meeting 11 Infectious individuals with a probability $r=0.1$ of being infected from each Infectious would generate an infection risk of 1.1 , which is impossible because an individual cannot be more than infected. 
To determine the cumulative risk, we instead have to calculate the probability of not being infected, which is $(1-r)$ per contact. The probability of not being infected in $N$ contacts is then $(1-r)^{N}$. The probability of not being infected during a time-step $(N=I \cdot m \cdot \Delta t)$ then becomes $(1-r)^{I \cdot m \cdot \Delta t}$. The expected number of infections per time-step, therefore, is $\mathrm{S} \cdot\left(1-(1-r)^{I \cdot m \cdot \Delta t}\right)$ which is approximately $S \cdot I \cdot r \cdot m \cdot \Delta t=S \cdot I \cdot p \cdot \Delta t$ when $r$ and $\Delta t$ are small. In the consistency proof in Section 5, the exact formula will be used, but for numerical calculations the $S \cdot I \cdot p \cdot \Delta t$ approximation is good, and can be used without problems. (End of comment)

The conceptual model created is thus a stochastic model of discrete individuals operating in continuous time.

Although time in the conceptual model is continuous, the use of a discrete time in the numerical models does not create any problems provided that the step-size $\Delta t$ can be made sufficiently short.

The following notation is used here: $\mathbf{S}, \mathbf{I}, \mathbf{R}$ stands for stage, while the number of individuals in a stage is denoted $S, I$ or $R$ (possibly with a time argument such as $S(t)$, etc.). Individuals are denoted $s, i$ or $r$ in accordance with their stage. $\mathbf{S} \rightarrow \mathbf{I} \rightarrow \mathbf{R}$ denotes the structure with transitions between stages.

\section{The micro-model}

The main idea of a SIR micro-model is to handle the complex composition of a heterogeneous population. However, here it is only used on a homogeneous population in order to investigate consistency between micro- and macro-modelling.

Implementation of the conceptual model as a micro-model is straight-forward. The micro-model is preferably implemented in a Discrete Event Simulation language or an object-orientated programming language but any programming language will suffice. The important thing is to describe the attributes of the individuals and their behaviours.

The only attributes are stage, which can take the values ' $\mathbf{S}$ ', 'I', 'R', the infection probability $p$, and the expected sojourn time $T$ in stage $\mathbf{I}$. The behaviour is described as transfers from stage $\mathbf{S}$ to stage $\mathbf{I}$ and from stage $\mathbf{I}$ to stage $\mathbf{R}$, respectively. For every possible meeting between individuals in stages $\mathbf{S}$ and $\mathbf{I}$, a uniform random number is drawn and compared to $p$ to decide if it results in transfer of the Susceptible individual. Furthermore, when an individual enters into stage $\mathbf{I}$, a random number for the sojourn time in the stage is drawn from an exponential distribution with the parameter set to $T$.

The time mechanism can be that used in Discrete Event Simulation [18], but for technical reasons (e.g., to avoid thinning $[19,20]$ to handle varying intensities in the infection intensity) it is more practical to use the classical updating procedure: Time $=$ Time $+\Delta t$, where $\Delta t$ is a small time-step. A micro-model then has the following structure in a pseudo code:

Generate $S$ entities in stage $\mathbf{S}$

Generate $I$ entities in stage $\mathbf{I}$ and randomise their TimeToLeaveI Again:

For each $s \in \mathbf{S}$

For each $i \in \mathbf{I}$ 


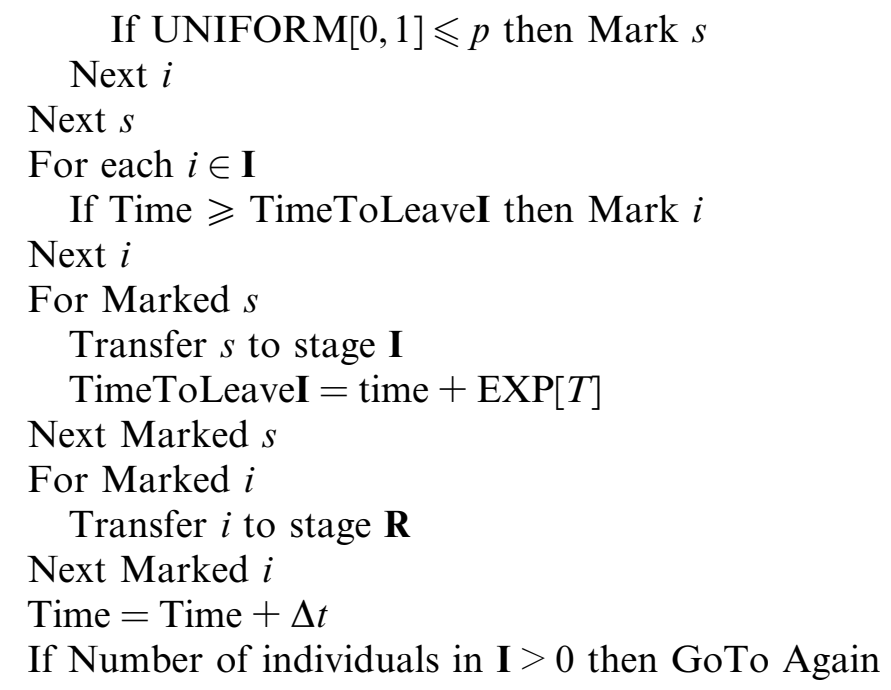

Here UNIFORM $[0,1]$ is a call to a generator of uniformly distributed random numbers between zero and one and $\operatorname{EXP}[T]$ is a call to a generator of exponentially distributed random numbers with the average $T$.

The micro-model agrees in every detail with the conceptual model. Note in particular that it uses stochastics on discrete individuals operating in almost continuous time provided that the time-step is sufficiently small.

\section{The macro-model}

A macro-model is an aggregation of the individuals in a stage into a single number hold by a state variable. This reduction of complexity means that there is no longer possible to refer to a specific individual in the macro-model.

The construction of the macro-model is performed in two steps. In the first step we build a deterministic differential equation model that acts on continuous fractions of the population. In step two we demonstrate how this model can be revised into a stochastic differential equation model acting on integer numbers of individuals.

\subsection{A deterministic macro-model}

Disregarding the stochasticity and the integer property of the individuals, we start with the classical SIR model [14], which is based on deterministic fractions of the population. This gives us the opportunity to demonstrate how stochastics and discreteness are implemented using the Poisson Simulation approach in a following step.

Let $S, I$ and $R$ stand for numbers of individuals in the consecutive stages $\mathbf{S}, \mathbf{I}$, and $\mathbf{R}$. Furthermore, let the probability $p$ and the expected sojourn time $T$ keep their values as deterministic quantities instead of being parameters to stochastic distributions. We then get the classical, deterministic SIR model $[1,2,5]$ as: 


$$
\mathrm{d} S / \mathrm{d} t=-p \cdot S \cdot I, \mathrm{~d} I / \mathrm{d} t=p \cdot S \cdot I-I / T, \mathrm{~d} R / \mathrm{d} t=I / T .
$$

Using the Euler algorithm, we can rewrite the model as difference equations:

$$
\left\{\begin{array}{l}
S(t+\Delta t)=S(t)-\Delta t \cdot F_{1}(t) \\
I(t+\Delta t)=I(t)+\Delta t \cdot F_{1}(t)-\Delta t \cdot F_{2}(t) \\
R(t+\Delta t)=R(t)+\Delta t \cdot F_{2}(t) \\
F_{1}(t)=S(t) \cdot I(t) \cdot p \\
F_{2}(t)=I(t) / T
\end{array}\right.
$$

The macro-model differs from the conceptual model in that it is based on deterministic fractions of individuals instead of stochastics on discrete individuals. Like the micro-model, it operates in almost continuous time.

To replace the deterministic mechanism transferring fractions of individuals between stages during a time-step by a stochastic mechanism operating on integer numbers of individuals, we first briefly present the mathematical construction of the Poisson Simulation approach.

\subsection{Poisson Simulation}

Poisson Simulation is a general method within the Continuous System Simulation concept for modelling randomness in a dynamic context as opposed to just adding noise. It has some important characteristics and features:

(1) A system of differential equations can be described in terms of states and flows. Then, a change in a state value only occurs through inflows and outflows to that state. In Poisson Simulation, the stochastics are located in the flow rates and not in states or parameters.

(2) The stochastics are implemented so that the integration step-size $\Delta t$ can be adjusted to the dynamic needs without distorting the model (just as it can in Continuous System Simulation).

(3) When the states are initiated to integer values, the Poisson mechanism adds or removes integer numbers, so the states remain integers over time.

In this section the mathematical foundations of Poisson Simulation are presented. This formulation both explains how the deterministic macro-model can be reformulated into a stochastic model and subsequently forms the basis of proof for the full consistency between the stochastic micro and macro approaches.

The Poisson process is the foundation of Poisson Simulation, but the latter is a much more flexible device since it incorporates intensities that can vary dynamically within the concepts of CSS modelling. The fundamental idea is that during a sufficiently short time-step, each intensity can be considered constant and can therefore be modelled by a Poisson process. The simulation can then be regarded as a sequence of Poisson processes. Furthermore, the number of events during the time-step is realised in CSS by drawing a Poisson distributed random number of the form $\operatorname{Po}[\Delta t \cdot$ intensity]. The mathematical construction of Poisson Simulation is thus deduced from the Poisson process as follows. 
A Poisson process is defined in the following way:

Let $E(t), t \geqslant 0$ be the number of times an event occurs in the time interval $(0, t)$. If the stochastic process $\{E(t), t \geqslant 0\}$ has the properties:

(1) The process has independent increments,

(2) $P[$ an event occurs exactly once in the interval $(t, t+h)]=\lambda \cdot h+o(h)$,

(3) $P[$ an event occurs more than once in the interval $(t, t+h)]=o(h)$,

then the process is a Poisson process with intensity $\lambda$.

A Poisson process requires a constant intensity, $\lambda$. In a dynamic relationship of the form $\mathrm{d} x / \mathrm{d} t=\lambda(x), \lambda(x)$ is an intensity function that depends on $x$ because of feedback. Therefore, a stochastic differential equation cannot be based directly on a Poisson process. However, the Euler difference equation $x(t+\Delta t)=x(t)+\Delta t \cdot \lambda(x(t))$ corresponding to $\mathrm{d} x / \mathrm{d} t=\lambda(x)$ can be calculated step-wise with $x(t)$ fixed in the time interval $(t, t+\Delta t)$. Since the difference equation during this time interval has a fixed intensity, we can include stochastics here as a Poisson process.

Using this idea, the number of events (e.g., the number of individuals changing their stage) during a very short time interval $(t, t+\delta t)$, where $\delta t \ll \Delta t$, follows the requirements:

(1) The process has independent increments,

(2) $P[$ an event occurs exactly once in the interval $(t, t+\delta t)]=\lambda \cdot \delta t+o(\delta t)$,

(3) $P[$ an event occurs more than once in the interval $(t, t+\delta t)]=o(\delta t)$,

and is therefore a Poisson process with intensity $\lambda$ (as long as $\lambda$ is constant; i.e., during $(t, t+\Delta t)$ ).

According to a fundamental theorem [21], the number of events $E$ during the interval $(t, t+\Delta t)$ where $\lambda$ is given a constant value, is then Poisson distributed with the expected value $\Delta t \cdot \lambda$; i.e., $E(t, t+\Delta t) \in \operatorname{Po}(\Delta t \cdot \lambda)$.

Poisson Simulation is based on this result and expresses a replication (a simulation run) of the stochastic difference equation

$$
X(t+\Delta t)=X(t)+P o[\Delta t \cdot \lambda(X(t))],
$$

as a sequence of Poisson processes defined on $[0, \Delta t),[\Delta t, 2 \Delta t),[2 \Delta t, 3 \Delta t), \ldots,[(\mathrm{N}-1) \Delta t, N \cdot \Delta t)$; where $N \cdot \Delta t$ is the length of the simulation (where $\left[t_{1}, t_{2}\right.$ ) means $t_{1} \leqslant$ time $<t_{2}$ ). Thus, Poisson Simulation of a stochastic variable is defined as:

$$
\sum_{i=1}^{N} \text { Poisson_process }(i)=\sum_{i=1}^{N} \operatorname{Po}\left[\Delta t \cdot \lambda_{i}\right] ; \text { where the interval } i \text { is }[(i-1) \cdot \Delta t, i \cdot \Delta t) .
$$

Thus, a stochastic system of difference equations corresponding to those in Section 4.1 can be obtained using Poisson distributions in the flow equations to express the number of events during each time-step. This reformulation of the deterministic macro-model is performed in the following section.

\subsection{Making the macro-model stochastic and discrete}

The deterministic and fractional SIR macro-model in Section 4.1 can now be reformulated into a stochastic approach using the Poisson Simulation technique mathematically defined 
above. This means that the fractional transitions during a time-step: $\Delta t \cdot F_{1}$ and $\Delta t \cdot F_{2}$ (where $F_{1}$ and $F_{2}$ are intensities) are replaced by a stochastic mechanism based on the Poisson distribution.

Since we have independency between the stage-changing events at a certain short interval in time, the number of transferred cases during $t$ to $t+\Delta t$ must be Poisson distributed with the expected values $\Delta t \cdot F_{1}$ and $\Delta t \cdot F_{2}$, respectively, as arguments. The stochastic macro-model thus takes the form:

$$
\left\{\begin{array}{l}
S(t+\Delta t)=S(t)-\Delta t \cdot F_{1}(t) \\
I(t+\Delta t)=I(t)+\Delta t \cdot F_{1}(t)-\Delta t \cdot F_{2}(t) \\
R(t+\Delta t)=R(t)+\Delta t \cdot F_{2}(t) \\
F_{1}(t)=P o[\Delta t \cdot S(t) \cdot I(t) \cdot p] / \Delta t \\
F_{2}(t)=P o[\Delta t \cdot I(t) / T] / \Delta t .
\end{array}\right.
$$

(Or with $F_{1}(t)=P o\left[S(t) \cdot\left(1-(1-r)^{I(t) \cdot m \cdot \Delta t}\right)\right] / \Delta t$ to be exact (see Comment in Section 2). This form will be used in the consistency proof in Section 5, below.)

Above, $P o[\cdot]$ means that a random number is drawn from a Poisson distribution at each timestep.

Note that since $P o[\cdot]$ has integer outcomes, the states $S, I$ and $R$ stay integer (provided that they are initiated to integer values). Poisson Simulation thus provides a stochastic macro-model operating on integer entities.

This is perhaps an appropriate place for a warning about two common mistakes. First, even though two or more inflows (or outflows) may be merged in accordance with $\operatorname{Po}\left[\Delta t \cdot \lambda_{1}\right]+$ $\operatorname{Po}\left[\Delta t \cdot \lambda_{2}\right]=P o\left[\Delta t \cdot\left(\lambda_{1}+\lambda_{2}\right)\right]$, it seriously distorts the model to use this on differences because: $\operatorname{Po}\left[\Delta t \cdot \lambda_{1}\right]-P o\left[\Delta t \cdot \lambda_{2}\right] \neq \operatorname{Po}\left[\Delta t \cdot\left(\lambda_{1}-\lambda_{2}\right)\right]$. Second, what comes out of stage $\mathbf{S}$ is identical to what goes into stage $\mathbf{I}$. These quantities cannot be made independent by writing: $S(t+\Delta t)=S(t)-P o\left[\Delta t \cdot F_{1}\right]$ and $I(t+\Delta t)=I(t)+P o\left[\Delta t \cdot F_{1}\right]-P o\left[\Delta t \cdot F_{2}\right]$, because then two independent $F_{1}$ flows would be obtained from two separate Poisson processes with the same intensity $\Delta t \cdot F_{1}$.

Poisson Simulation is a theoretically sound, easy handled and computer efficient way of realising a stochastic population model in a macro setting. The calculation effort is also almost independent of the size of the population. It is easily applied in any Continuous System Simulation language where a random number generator for the Poisson distribution is included. It is also easy to write the model directly in a general purpose programming language. A good Poisson random number generator can be found in, e.g., Numerical Recipes [22]. Poisson Simulation is presented in $[11,23,24]$. It is also convenient to have a supervisory programme that can order a number of simulations, collect the outcomes, analyse them and present the results in statistical terms. Such programmes [25,26] are available for Powersim [27] and for MATLAB [28].

Finally, note that the deterministic model is embedded within the stochastic macro-model. Just remove the $\operatorname{Po}[\Delta t \cdot] / \Delta t$ part of the flow rate equations $F_{1}$ and $F_{2}$, above. The concept 'embedded model' is important because when an embedded deterministic model behaves similarly to the stochastic model, it is meaningful to perform theoretical analysis on the simpler, deterministic model [5]. 


\section{Consistency between micro- and macro-models}

For both the micro-model and the stochastic macro-model, the following is valid:

- The structures: $\mathbf{S} \rightarrow \mathbf{I} \rightarrow \mathbf{R}$ are the same.

- The initial values $S(0), I(0)$ and $R(0)$ are integers and have the same values in both models.

- The parameters, $p$ and $T$ have the same values and the time-step $\Delta t$ is also the same.

- Changes of stage only occur through events; as individual transfers or as aggregated number of events in the flow rates $F_{1}$ and $F_{2}$. Furthermore, these events during the short time intervals $(t, t+\Delta t)$ are independent.

Therefore, it is sufficient to show that the transfer mechanisms, $\mathbf{S} \rightarrow \mathbf{I}$ and $\mathbf{I} \rightarrow \mathbf{R}$, are equivalent, i.e., do the same job, despite their different realisations. In particular, the sojourn time (activity) should have the same statistical distribution.

- For $\mathbf{S} \rightarrow \mathbf{I}$, the conceptual model states that for each time unit there is a probability $p$ of the event of a meeting between a given Susceptible and a given Infectious individual where the infection is transmitted. With $S$ Susceptible individuals and $I$ Infectious individuals there are $S \cdot I$ possible meetings.

In the micro-model, the probability of every Susceptible becoming infected by an Infectious individual is implemented in a straight-forward way as shown in Section 3.

Since a Susceptible individual can only be infected once, this implies that his/her risk per time unit of infection from $N$ meetings with Infectious individuals is $\left(1-(1-r)^{N}\right)$. During the short time-step $\Delta t$ the risk of each Susceptible individual being infected is then $1-(1-r)^{I \cdot m \cdot \Delta t}$. Since the risks of infection of the $S$ Susceptible individuals are independent, the number of infections during $\Delta t$ becomes $P o\left[S \cdot\left(1-(1-r)^{I \cdot m \cdot \Delta t}\right)\right]$ distributed, which was used for the macro-model.

The micro- and macro- mechanisms for $\mathbf{S} \rightarrow \mathbf{I}$ are therefore consistent with the conceptual model and numerically equivalent when using the same step-size, $\Delta t$.

- The transfer $\mathbf{I} \rightarrow \mathbf{R}$ constitutes the end event of the sojourn time activity of the Infectious stage. For the duration in stage $\mathbf{I}$ the conceptual model assumes an exponential distribution. This is directly implemented at individual level in the micro-model. At time $t_{1}$, defining the arrival of an individual to stage $\mathbf{I}$, the time $t_{2}$ for departure is drawn from an exponential distribution: $t_{2}=t_{1}+\operatorname{Exp}[T]$.

To show that this is equivalent to the $\mathbf{I} \rightarrow \mathbf{R}$ Poisson mechanism in the macro-model, we utilise the fact that the departure time for an individual is independent of the arrival time. The former is a consequence of the exponential distribution being memoryless. This means that if the individual at time $t$ is in stage $\mathbf{I}$, the expected time until departure is $T$, independent of when the individual arrived in stage $\mathbf{I}$ and independent of time $t$, as long as departure has not yet occurred. (To illustrate this, consider a radioactive atom with the expected time until decay, $T$. Independent of how long this atom has existed, the expected time from now to its decay is still $T$.) Since the probability of the individual departing during $(t, t+\Delta t)$ is independent of its arrival time (under the condition that the individual has not already departed), the probability of departing is proportional to $\Delta t$ and to $1 / T$; i.e., to $\Delta t / T$. For $I$ individuals aggregated in the 
stage, the departure intensity is $I / T$. Because of the independence of the departures, the conditions for a Poisson process with intensity $I / T$ are fulfilled, so the number of departures from stage $\mathbf{I}$ to stage $\mathbf{R}$ during $(t, t+\Delta t)$ is $P o[\Delta t \cdot I / T]$, which is used in the macro-model.

An alternative proof is obtained by starting from the aggregated macro-model and disaggregating it to end up in the $\mathbf{I} \rightarrow \mathbf{R}$ construction of the individually based micro-model. Then we consider the $\Delta t \cdot F_{2}=P o[\Delta t \cdot I / T]$ departures during $(t, t+\Delta t)$ (from the last equation in $(*)$ in Section 4.3). First, $P o[\Delta t \cdot I / T]$ can be disaggregated into $I$ terms, because

$$
\operatorname{Po}[\Delta t \cdot I / T]=\operatorname{Po}[\Delta t \cdot 1 / T]+\operatorname{Po}[\Delta t \cdot 1 / T]+\ldots+\operatorname{Po}[\Delta t \cdot 1 / T],
$$

reflectingthe $I$ individuals. Second, the Poisson distribution of the number of departures during a time interval in the macro-model implies an exponential distribution for the expected time to departure in the micro-model.

To show this, we consider one Infectious individual so $P o[\Delta t \cdot 1 / T]$ is the probability of its departure during the interval $\Delta t$. The Poisson distribution for zero or one departure is then: $p(k)=\mathrm{e}^{-m} \cdot m^{k} / k !$ where $k=0,1($ and $m=\Delta t \cdot 1 / T)$.

To show that this implies an $\exp [T]$ distribution for the sojourn time of an Infectious individual, we ask when the departure from now $=t$ will occur? Setting $t=0$ for simplicity and defining the first departure point in time $\tau$, this departure event, $D$, occurs if and only if the departure has not occurred during the interval $(0, \tau)$, i.e., if the number of departures at $\tau$ is zero; where this number $\in \operatorname{Po}(\lambda \cdot \tau)$, and $\lambda=1 / T$. Since the Poisson distribution is defined by

$$
p(k)=\mathrm{e}^{-m} \cdot m^{k} / k !,
$$

the probability of $k=0$ events before $\tau$ is $\mathrm{e}^{-m}=\mathrm{e}^{-\lambda \cdot \tau}$, i.e., $P(D>\tau)=\mathrm{e}^{-\lambda \cdot \tau}$. The complement to the probability of no departure is $P(D \leqslant \tau)=1-\mathrm{e}^{-\lambda \cdot \tau}$. However, $P(D \leqslant \tau)$ is the distribution function $F_{\mathrm{D}}(\tau)=1-\mathrm{e}^{-\lambda \cdot \tau}$, so $D$ must have exponential density distribution $\mathrm{e}^{-\lambda \cdot \tau}=\mathrm{e}^{-\tau / T}$. Thus, the sojourn time has an $\exp [T]$ distribution, just as is used in the micro-model.

Thus, the conceptual SIR model and its micro and macro realisations are mutually consistent. We can so easily prove the consistency because we assumed the sojourn time distribution in stage I to be exponential. For other time distributions, we could not directly relate the sojourn time to a departure event independent of the arrival time.

However, for sojourn time distributions in the conceptual model and micro-models other than exponential types, the consistency with the macro-model can also be preserved. This is because the sojourn time distribution of the macro-model can be extended to any time distribution that can be constructed by dividing the stage into a series of $n$ sub-stages, e.g., $\Gamma(n, T / n)$ [13]. Furthermore, any distribution, that can be approximated using sub-stages in series and/or parallel and with the same or different time parameter values of the sub-stages, can be used while keeping the equivalence between the micro and macro approaches.

\section{Testing the micro- and macro-models}

For a uniquely defined conceptual model, the outcomes of different, correctly formulated simulation models should be consistent, irrespective of their technical formulations. Since the results from a stochastic model are probability distribution functions (pdf), this means that the 
micro- and macro-models should produce identical probability distribution functions for the outcome. To test this, the stochastic, individual micro-model and the stochastic, aggregated macromodel were simulated using the following parameter values:

$$
S(0)=1000, I(0)=1, R(0)=0 \text { individuals, }
$$

$p=0.0003$ per individual and time unit, $T=4$ time units and $\Delta t=0.1$ time units.

(This implies a basic reproduction number $R_{0}=p \cdot S \cdot T=1.2$.)

To demonstrate what can happen for a non-consistent model, we also include the embedded deterministic macro-model with the same parameter values. The models shown in Sections 3, 4.1 and 4.3 were programmed in Turbo Pascal [29] and the random number generator used was 'ran3' as presented in [22].

Table 1 shows the results of 10000 replications using the micro-model and the stochastic macromodel and one simulation of the embedded deterministic macro-model. The outputs studied are the number of Susceptible individuals becoming infected during the epidemic, $S(0)-S($ End), and the length of the epidemic (until $I$ became zero).

\subsection{The stochastic models}

The average number of Susceptible individuals becoming infected during the epidemic for the micro-model falls within the $95 \%$ confidence interval of that for the macro-model and vice versa. The same applies for the average length of the epidemics.

Poisson Simulation not only gives consistent estimates of the average number of infected individuals and the average length of the epidemic, but also gives the full statistical outcome in terms of pdfs, from which one can derive all kinds of statistics such as average, variations, skewness and confidence intervals. The pdfs from the micro- and macro-models are compared in Fig. 2.

As seen from Fig. 2, the overall shapes of the probability density functions are very similar. When comparing pairs of micro/macro bars some variations can be seen, especially for the smaller bars with values around $0.005-0.01$ (representing 50-100 replications). For each bar we expect a $\operatorname{Bin}(n, p r)$ distribution for the $n=10000$ replications. The actual outcome behind each bar then suggests $n \cdot p r \approx 50-100$ replications, giving $p r \approx 0.005-0.01$. The expected standard deviation then becomes $\sqrt{n \cdot p r(1-p r)}$, which is about $7-10$ replications, implying a 95\% confidence interval of about \pm 14 to \pm 20 . Taking into account that the outcomes of the two bars in a pair are independent, we expect that the difference between them should be within 20-28 replications or $20 / 50=40 \%$ and $28 / 100=28 \%$ of the outcome in $95 \%$ of the pairs of size $0.005-0.01$. This agrees well with what we see in Fig. 3.

Table 1

Results obtained using the micro-model, the stochastic macro-model and the embedded deterministic macro-model

\begin{tabular}{lllll}
\hline Model & $\begin{array}{l}\text { Average } S(0)-S(\text { End }) \\
(95 \% \text { C.I. })\end{array}$ & Min \& Max & $\begin{array}{l}\text { Average length of } \\
\text { epidemic }(95 \% \text { C.I. })\end{array}$ & Min \& Max \\
\hline Micro & $54.6(52.2-57.0)$ & $0 \& 579$ & $22.5(21.7-23.2)$ & $0.1 \& 247.6$ \\
Stochastic macro & $54.5(52.1-56.9)$ & $0 \& 560$ & $21.9(21.1-22.6)$ & $0.1 \& 223.4$ \\
Deterministic macro & 318.5 & 318.5 & $\infty(207.4$ for 1 case left $)$ & $\infty$ \\
\hline
\end{tabular}




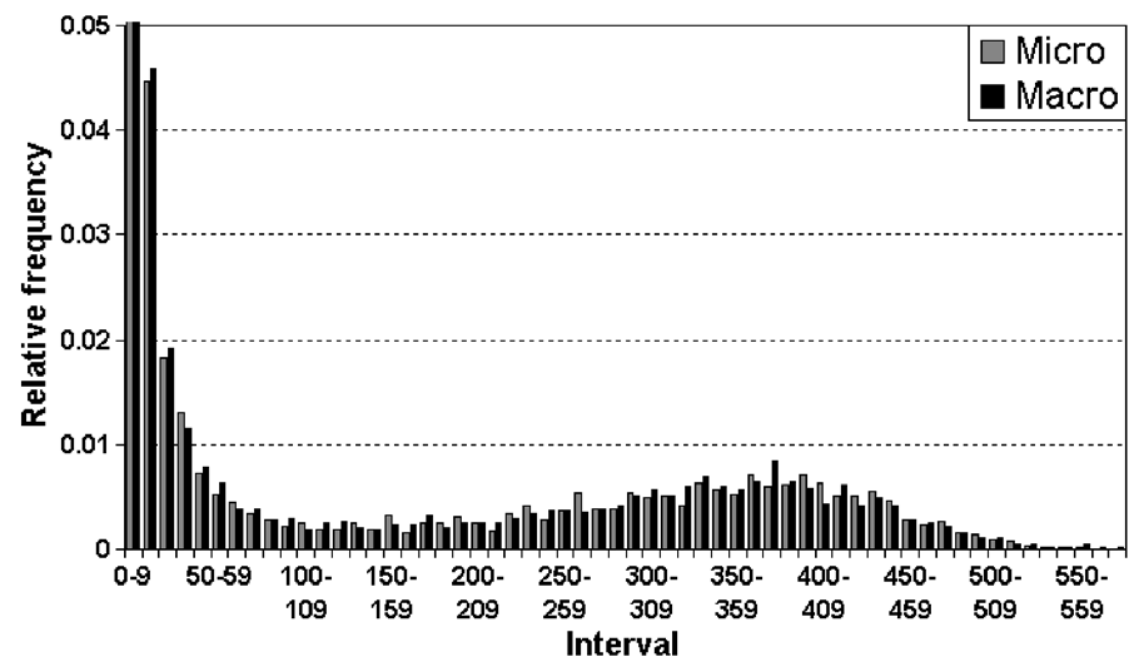

Fig. 2. Comparison of the pdfs of the number of Susceptible individuals becoming infected during the epidemic $[S(0)-$ $S$ (End)] as calculated from 10000 replications of the micro-model and of the stochastic macro-model with the parameter values $S(0)=1000, I(0)=1, R(0)=0, p=0.0003$ and $T=4$. The bar intervals are $0-9,10-19, \ldots, 560-579$. (The first pair of bars (0-9) has the values 0.7388 and 0.7422 for the micro- and macro-models, respectively.)

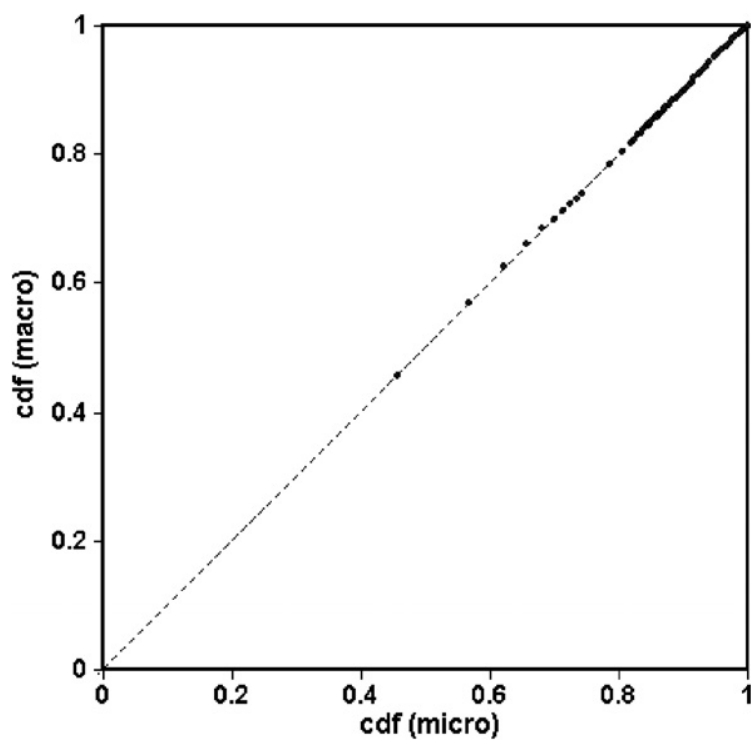

Fig. 3. $p-p$ plot of the cdfs of the number of Susceptible individuals becoming infected during the epidemic from the micro- and stochastic macro-simulations. (The cdfs for $\leqslant 0, \leqslant 1, \leqslant 2, \ldots, \leqslant 9, \leqslant 19, \leqslant 29, \ldots, \leqslant 579$ Susceptible individuals becoming infected are plotted.)

In order to better compare the outcomes, we focus on the cumulative distribution functions (cdf). We thereby eliminate the variations because of small numbers without losing any information. Instead of doing this in a straight-forward way, obtaining two curves, we use a probability- 
probability plot ( $p-p$ plot) [19]. The $p-p$ plot is a graph of the micro cdf against the macro cdf. For example, the micro cdf of zero infected cases is 0.4555 and the corresponding macro cdf is 0.4563 , giving the point $(0.4555,0.4563)$. The second point representing $\leqslant 1$ case gets the coordinates $(0.5692,0.5663)$, etc.

A perfect match between the models would result in a straight diagonal line between $(0,0)$ and $(1,1)$. Any deviation from that line is easily detected by the eye. As one can see, the $p-p$ plot in Fig. 3 shows an almost perfect alignment to the diagonal.

\subsection{The deterministic model}

To demonstrate the drastic effects of a similar (and often used!) not fully consistent model, we also compare with the embedded deterministic macro-model.

The results in Table 1 show that most of the replications with the stochastic models give no epidemic. The average numbers contracting the disease are 54.6 and 54.5 , compared with the categorical result of 318.5 for the deterministic model, i.e., an approximately six-fold difference. The average lengths of the epidemics display even less consistent results.

The remarkable fact is that there is no similarity between the results from the deterministic and those from the stochastic models on any point. The results are not even close.

Although dynamic models of epidemics have a long tradition going back to Kermack and McKendrick in 1927 [14] and Greenwood in 1931 [30], a number of methods for handling the stochastics have been proposed since then $[1,15]$. These models are usually classified as continuous time or discrete-time models rather than macro- or micro-models and usually focus on analysis rather than simulation. A discussion of discrete-time models is found in Appendix B.

Macro-models based on differential or difference equations and stochastics are often introduced in some additive way. With the introduction of computers and Discrete Event Simulation programmes, it became possible to directly formulate the conditions for the individual in micro-models and obtain a technically correct micro-model of a conceptual model.

Although comprehensive text books with thorough discussions of deterministic and stochastic models such as [15] are available, many modern textbooks on epidemics still present only deterministic models and many contemporary papers base their studies on deterministic SIR/SEIR macro-models - probably because of lack of knowledge on how to include the stochastics. The results from such studies therefore lack consistency with the results from micro studies and from the intensions of the underlying conceptual model.

\section{Summary and discussion}

In this section the content and findings of the paper are first summarised, with the focus on how the Poisson Simulation technique can be defined and used for population models to construct correct macro-models consistent with micro-models. Thereafter, the nature of stochastics and dynamics in a population model is discussed and a general form for macro population models is presented. Possible extensions beyond the class of population models are then presented. In three appendices we broaden the scope. In Appendix A, some other population models are given 
in the Poisson Simulation form. In Appendix B we investigate what would happen if discrete-time modelling were used instead of continuous. Finally, in Appendix C the Markov approach, which also is a consistent way to describe population models, is discussed and compared with Poisson Simulation.

\subsection{Summary}

The main objective of this work is to demonstrate a method to achieve consistency (in terms of containing no contradictions) between micro- and macro-models of populations evolving in continuous time.

With Poisson Simulation, a new method for aggregated modelling of population models was introduced [11]. By defining Poisson Simulation in strictly mathematical terms as a series of Poisson processes that can be summed up into sequences of Poisson distributions with dynamically varying parameters, we were able to prove the logical consistency between a micro- and a macro-model realisation of the same conceptual population model. This was demonstrated on a SIR epidemic model containing both the event and activity aspects described earlier.

Two logically consistent models should produce consistent results, and we illustrated this by making 10000 replications each of the micro- and macro-models.

Simulation of the models also gave the opportunity to demonstrate the drastic consequences when consistency is lost. An example of this is obtained when the conceptual SIR model is realised as the deterministic fractional model embedded in the stochastic macro-model. The result was that the epidemic never ceased and that the number of infected individuals became six times larger than the average from a correct model.

Any extension of the SIR model in terms of more states/stages can easily be realised in microor macro-modelling. If the conceptual model assumes statistical distributions other than the memoryless exponential distribution for the time duration of an activity, this poses no problem for the micro-modelling. For the macro-model, good approximations of such activities can be achieved by structures of states in series and/or parallel. Special constructions within the macro-modelling approach, discussed in [24], can also be used for this purpose.

The SIR model was only chosen as a demonstrative example. Any population model such as models of radioactive decay [11,31], biological competition [23,31,32], predation (e.g., Lotka-Volterra) [11,31-33], demographical development [11,31], Gompertz' growth models [23,31,32,34], epidemic processes $[1,15]$, case-control studies $[23,35]$, ion channels in a neurone [23,36], models of warfare (like Lanchester's model of warfare) $[23,31,37,38]$, etc. can utilise the Poisson Simulation approach to develop a consistent macro-realisation, see Appendix A. In [11] there is an example of logistic and Volterra models and in [24] it is shown how this approach can be used to include queuing problems in a macro-setting. In [24], a section discusses in general terms the close relationship between Poisson Simulation and Discrete Event Simulation (or micro-simulation), despite large differences in the technical realisations.

A number of phenomena distinguish the behaviour of stochastic dynamic population models from that of deterministic models. For example, very different behaviours, results and statistical estimates can be obtained. New phenomena such as extinction may also occur in a stochastic model, while a deterministic model can recover from a small fraction of an individual. Furthermore, new qualities such as oscillations may be excited by the stochastic variations. Correct 
modelling of dynamics and stochastics is therefore crucial, and Poisson Simulation is a powerful technique to handle this for population models.

\subsection{A general form for population models}

It is a more or less implicit opinion that stochastics should be avoided in Continuous System Simulation. This is based on the idea that stochastics add additional noise that only obscures the results and forces the analyst to run many replications to get precision in the estimates. Therefore, textbooks on CSS usually avoid the subject or mention it very briefly and CSS languages usually provide only a few fundamental distributions for random deviates.

Old traditions that dynamics are handled by differential equations and stochastics by a form of random variation nurture the misconception that a stochastic and dynamic model is composed of differentials and probabilities as unrelated components. However, stochastics is not a phenomenon to be simply added (or multiplied) to the dynamic behaviour (except for very special cases such as signal + noise in a radio receiver). For a population model, stochastics and dynamics are only different aspect of changes within a system or model. This is visible in micro-model simulation where a change/event is the only mechanism and it brings a certain impact on the following development over time, irrespective of whether the event is described as deterministic or stochastic in its size or its time of appearance. While the general microdescription of a population only refers to events (and activities), the macro-description refers to numbers of events in short intervals of time.

All the population models mentioned in Section 7.1, as well as all population models found in the literature, turn out to have the same general macro form given by:

$$
\mathrm{d} x_{k}=P o\left[\mathrm{~d} t \cdot \lambda_{\text {in }}(\underline{x}, t)\right]-P o\left[\mathrm{~d} t \cdot \lambda_{\text {out }}(\underline{x}, t)\right] ; k=1,2 \ldots n,
$$

where $x_{k}$ denotes state $k$ and $\underline{x}$ is an $n$-dimensional vector, see Appendix A. (It is $x$ in the $\lambda$ argument that gives the feedback generating the dynamics. Removing this feedback results in a stochastic process which can be time-variable, $\lambda(t)$, or time-constant, $\lambda$.)

From the macro process, statistics can then be obtained by cumulating (integrating) over a time interval. Further, 'pure' dynamics is a special case when the population is very large. The law of large numbers then ensures that the variations are negligible compared with the number of individuals, so the equation above collapses into the embedded equation:

$$
\mathrm{d} x_{k}=\mathrm{d} t \cdot \lambda_{\text {in }}(\underline{x}, t)-\mathrm{d} t \cdot \lambda_{\text {out }}(\underline{x}, t),
$$

or $\mathrm{d} x_{k} / \mathrm{d} t=\lambda(\underline{x}, t)$. Note, however, that it is not sufficient that the total population is large. Subpopulations may also have to be. For example an influenza starts with a single infected individual and may spread within a very large population, but the development of the epidemic cannot be modelled deterministically although the total population is very large.

\subsection{Possible extensions}

This paper focuses on population models but the technique demonstrated goes beyond this class of models. For example the population part may be included in a larger, otherwise deterministic, model. This still works because the integer numbers of entities can affect continuous 
quantities (such as cost, weight, etc.) and continuous entities can affect the number of new or removed entities via the continuous argument of the $P o[\Delta t \cdot \arg ]$ construction. For example, in an extended SIR model the number of individuals hit by the disease can be assigned a cost and the number of individuals considering a vaccination may be a random function of the price of a vaccination such that $P o[\Delta t \cdot f($ vaccination_price $)]$.

Furthermore, the Poisson mechanism does not exclude other types of randomness. For example, an insurance company is interested in the number of accidents and their costs. The seasonal variations may be modelled by $P o[\Delta t \cdot \lambda(t)]$, while the cost of an accident is, e.g., exponentially distributed. Another example is digital telephone systems handling packages of information where the arrivals may be Poisson distributed (e.g., $P o[\Delta t \cdot \lambda(x, t)]$; where $x$ takes care of some correlation from another part of the system) and the length of a package varies in accordance to another distribution. However, this goes beyond the focus of this paper.

\section{Appendix A. A general form for Poisson Simulation of population models}

All the population models mentioned in Section 7.1, as well as all population models found in the literature, turn out to have the same general macro form given by:

$$
\mathrm{d} x_{k}=P o\left[\mathrm{~d} t \cdot \lambda_{\text {in }}(\underline{x}, t)\right]-P o\left[\mathrm{~d} t \cdot \lambda_{\text {out }}(\underline{x}, t)\right] ; k=1,2 \ldots n,
$$

where $x_{k}$ denotes state $k$ and $x$ is an $n$-dimensional vector. In numerical form we then have:

$$
\left\{\begin{array}{l}
x_{k}(t+\Delta t)=x_{k}(t)+\Delta t \cdot\left(F_{k \text { in }}-F_{k_{-} \text {out }}\right) ; \text { where } k=1 \ldots n . \\
F_{k \text { in }}=P o\left[\Delta t \cdot \lambda_{\text {in }}(\underline{x}, t)\right] / \Delta t \\
F_{k_{\text {out }}}=P o\left[\Delta t \cdot \lambda_{\text {out }}(\underline{x}, t)\right] / \Delta t .
\end{array}\right.
$$

Some examples:

$$
\begin{array}{ll}
\mathrm{d} x=-P o[\mathrm{~d} t \cdot a \cdot x] & \text { Radioactive decay model }[11,23] . \\
\mathrm{d} x=P o[\mathrm{~d} t \cdot a \cdot x]-P o\left[\mathrm{~d} t \cdot b \cdot x^{2}\right] & \text { Logistic model [23]. } \\
\mathrm{d} x_{1}=P o\left[\mathrm{~d} t \cdot a \cdot x_{1}\right]-P o\left[\mathrm{~d} t \cdot b \cdot x_{1} \cdot x_{2}\right] & \text { Lotka-Volterra model }[11,23] . \\
\mathrm{d} x_{2}=P o\left[\mathrm{~d} t \cdot c \cdot x_{1} \cdot x_{2}\right]-P o\left[\mathrm{~d} t \cdot \mathrm{d} \cdot x_{2}\right] & \\
\mathrm{d} x_{1}=-P o\left[\mathrm{~d} t \cdot a \cdot x_{2}\right] & \text { Lanchester's model of warfare [23]. } \\
\mathrm{d} x_{2}=-P o\left[\mathrm{~d} t \cdot b \cdot x_{1}\right] & \\
\mathrm{d} x_{1}=-P o\left[\mathrm{~d} t \cdot p \cdot x_{1} \cdot x_{2}\right] & \text { The SIR model in this paper. } \\
\mathrm{d} x_{2}=P o\left[\mathrm{~d} t \cdot p \cdot x_{1} \cdot x_{2}\right]-P o\left[\mathrm{~d} t \cdot b \cdot x_{2}\right] & \\
\mathrm{d} x_{3}=P o\left[\mathrm{~d} t \cdot b \cdot x_{2}\right] & \\
\mathrm{d} x=P o[\mathrm{~d} t \cdot \lambda]-\operatorname{MIN}(P o[\mathrm{~d} t \cdot \mu \cdot x], x) & \begin{array}{l}
M / M / \infty \text { queue model (where } x \text { is the actual number of } \\
\text { queuing and served individuals, } \lambda \text { is the arrival intensity }
\end{array} \\
& \begin{array}{l}
\text { and } 1 / \mu \text { is the mean service time). (MIN (arg1, arg2) takes } \\
\text { the smallest of its two arguments to prevent more } \\
\text { departures than the population in the queue. })[23,24] .
\end{array}
\end{array}
$$


See papers $[11,23,24]$ for a presentation of these and other population models treated by Poisson Simulation. The modeller should consult paper [11] for the underlying rules of Poisson Simulation. Note in particular that for models where what leaves one state $x_{i}$ enters another $x_{j}$, it is important that the numerical realisation uses a single flow rate equation as described in the warning note in Section 4.3.

Note also that the embedded models are easily obtained from the Poisson Simulation models.

\section{Appendix B. Discrete-time modelling}

This study is limited to continuous-time modelling. This seems reasonable since a population at study develops in continuous time. However discrete-time models such as Markov chain models [4,5], chain-binomial models [15], ARMA models [39], time series models [40], etc. are often used. Consistency with continuous-time models can then of course not be exactly achieved since continuous-time stochastics are based on the Poisson distribution while discrete-time stochastics, implying a sequence of random trials, are based on the binomial distribution.

Accepting the inconsistency of modelling continuous time by a sequence of discrete points, can macro- and micro-simulation models that are mutually consistent be constructed? Yes, in principle, but at the price of inflexible modelling, results that are functions of the time-interval between index points and a slower execution of the random parts.

In the very concept of discrete-time models there is no explicit time-step $\Delta t$ to be adjusted until it is small enough not to distort the dynamics. (Mathematically, the limes $\Delta t \rightarrow 0$ mechanism is lacking.) This cannot be introduced in a mathematically correct way without rebuilding the model and again fitting parameters and transition probabilities to system data. For continuous-time, the stochastic flow equations $\Delta t \cdot F=P o[\Delta t \cdot \lambda]$ can be freely adjusted with respect to $\Delta t$ without affecting the stochastics because of the Poisson property:

$$
\operatorname{Po}[\Delta t \cdot \lambda]=\operatorname{Po}[\Delta t \cdot \lambda / 2]+\operatorname{Po}[\Delta t \cdot \lambda / 2] \text {. }
$$

However $\operatorname{Bin}[n, \Delta t \cdot p] \neq \operatorname{Bin}[n, \Delta t \cdot p / 2]+\operatorname{Bin}[n, \Delta t \cdot p / 2]$, so such a time-scaling mechanism cannot even be introduced correctly in the discrete-time case.

Furthermore, discrete time implies that the macro- and micro-models must operate with the same index-time intervals to be consistent. (For continuous time models we only require the time-steps of the macro- and micro-models to be sufficiently small from a dynamic point of view without any need for them to be the same.) Even with this condition fulfilled results from discretetime models are arbitrary since parameters in the models, results and estimates are all coupled to the chosen time-interval between index points. Comparisons with models using other intervals cannot be made and results cannot be transferred freely between models since models using different intervals are inconsistent and thus produce inconsistent results and estimates.

Assuming that we were lucky enough to initially choose an interval between index points that was small enough from a dynamic point of view, we would then end up with a macro-model that produced almost correct result since $\operatorname{Bin}[n, \Delta t \cdot p] \rightarrow P o[n \cdot \Delta t \cdot p]$ when $\Delta t \cdot p \rightarrow 0$. However what we have then accomplished is just a model that executes considerably more slowly because each random sample from a $\operatorname{Bin}(n, \Delta t \cdot p)$ generator (using the direct method) requires $n$ calls to a uniform $U[0,1]$-generator which is much more than a direct Poisson generator requires $[6,19,20,22,41]$. (With the binomial approach each atom in a radioactive sample is tested for a pos- 
sible decay, each car for a possible accident or each person for a possible infection during every short time-interval $\Delta t$, while a Poisson approach only counts the number of decays, accidents or new infections during $\Delta t$.) Using a more sophisticated binomial algorithm would in best case detect that $\Delta t \cdot p$ is small enough to use a Poisson algorithm.

From a simulation point of view there seems to be no theoretical or practical advantage with describing continuous time as discrete using the two-parametric $\operatorname{Bin}[n, p]$-distribution instead of the one-parametric $P o[\lambda]$-distribution. A discrete-time approach is also technically inferior to constructing a stochastic Poisson Simulation model in a Continuous System Simulation language where practical tools such as time-step adjustment, random numbers of different distributions, a function library with various functions or even tailor-made table look-up functions, output facilities, etc. are at hand for the model building.

\section{Appendix C. Markov models and Poisson Simulation}

What makes Markov theory $[4,5]$ important in this context is that it is probably the most powerful method to theoretically analyse population models. For a given conceptual population model, realisations as a Markov model, micro-model and Poisson Simulation model are all consistent. This is of great importance because it provides consistent tools for theoretical analysis, as well as for numerical experiments on the same conceptual model. Markov modelling is also widely used for numerical studies. However, it has a number of disadvantages and limitations compared with Poisson Simulation.

\section{C.1. Discrete Markov process in continuous or discrete time}

A population model can be described as a discrete Markov process, also called Markov jump process, in discrete or continuous time [4,5]. However for sufficiently small time-steps in the discrete-time case, the result will be the same as for the continuous time case.

The first step in Markov modelling is to define the set of states in which the process can exist. For a population model this means all combinations of sub-population sizes in the model.

For a simple SIR model we have the $k=3$ sub-populations $\mathbf{S}$, I and $\mathbf{R}$. Since $S+I+R=m$, we have two degrees of freedom because $\mathbf{R}$ contains the remaining $m-S-I$ individuals.

For example, if we have only $m=3$ individuals in a simple SIR model, these 3 individuals can be in stages $\mathbf{S}, \mathbf{I}$ or $\mathbf{R}$ according to the 10 states $\{(3,0,0),(2,1,0),(2,0,1),(1,2,0),(1,1,1),(1,0,2)$, $(0,3,0),(0,2,1),(0,1,2),(0,0,3)\}$. The general formula for the number of states in the state-space is: $\left(\begin{array}{c}m+k-1 \\ k-1\end{array}\right)$, where $m$ is the number of individuals in the population and $k$ is the number of sub-populations [5]. Already for $m=100$ individuals, a simple SIR model has a state-space of 5151 states, and the SIR model of a small village with $m=1001$ inhabitants (the model in this paper) gives a huge Markov model of about half a million states (instead of three state variables in a Poisson Simulation model).

For a SIR model where the duration of the infectious stage has a $\Gamma(3, \beta)$ distribution (i.e., three exponential sub-stages in a series), the model will have the stages $S, I 1, I 2, I 3$ and $R$. We then have $k=5$ and $m=10001$, so the number of elements in the state-space becomes about 40000000000 
for the Markov model. (Still this makes a small Poisson Simulation model of only five state variables which we build in a few minutes and make 10000 replications from in less than ten minutes on an $2.4 \mathrm{GHz}$ PC.)

In the second step the transition probabilities between the states are described. (These have the form: $P\left[E_{i} \rightarrow E_{j}\right.$ in $\left.t, t+h\right]=a_{i j} h+o(h)$ which constitute the same starting-point as for the Poisson process from where Poisson Simulation is deduced. This is the reason why Markov models and Poisson Simulation models are consistent.)

The transition probabilities (which obey the Markov property that the new state of the process depends only on the present and all previous history is forgotten) are placed in a transition matrix (in the time discrete case) by which the new state of the process is updated time-step by time-step, or in an intensity matrix and calculated by the Kolmogorov differential equation in the continuous time case.

\section{C.2. Comparison of Markov and Poisson Simulation}

When it comes to simulation (numerical solving by many replications of a stochastic model) the Markov approach is more problematic. Here the Poisson Simulation approach has a number of advantages over Markov Simulation. From a numerical point of view a Markov model is a micro-model since there is no aggregation over state-space or time and every single transfer is modelled and calculated (but it differs from other micro-models in that the individuals are not distinguishable).

The main difference is that in Markov theory all possible states of a system are modelled while the Poisson Simulation approach aggregates over state-space and time. The aggregation over state-space means that the individuals are accumulated into a few state variables, where one state variable can represent any number of individuals.

The aggregation over time means that we can accept many transitions during a time-step $\Delta t$ instead of one or zero during a very small time-step $h$.

The advantages of Poisson Simulation compared to Markov Simulation are:

(1) Aggregation over state-space. For a Markov model the number of elements in the state-space grows combinatoricaly with the population size and the number of sub-populations, while the number of state variables in Poisson Simulation is small and independent of population size. What is a huge Markov model becomes a very small one in Poisson Simulation. Therefore, the Poisson Simulation model is much easier to build and gives a more lucid and comprehensive model.

Furthermore, the SIR model used here as an example referred to a limited population. For population models in general including growth of a population the number of states is unknown, which is a problem. If the model is to be run, e.g., 10000 times, the largest (a priori unknown) population has to be allocated for in the Markov matrix - or some dynamic expansions of the model have to be included when needed. In Poisson Simulation the state variables can take any number, so this is never a problem.

(2) The aggregation over time means that we can accept many transitions during a time-step $\Delta t$ (only limited by the time constants of the dynamics) instead of a very small time-step $h$ in the Markov model that is small enough to 'guarantee' that not more than one transition may occur in $(t, t+h)$. This aggregation reduces computation time to a small fraction. 
(3) The use of random number generators. In a Markov Simulation an event might happen during the next time-step. However, to conform with the $P\left[E_{i} \rightarrow E_{j}\right.$ in $\left.t, t+h\right]=a_{i j} h+o(h)$ concept, the time-step $h$ must be short enough not to risk more than one event. If, for example, $h$ is short enough to only erroneously give two events at one time in a hundred (not good!) then it will (because of independence) create one event only in about one case out of ten and zero events in about nine cases out of ten! This means that the number of calls to the random number generator (RNG) is ten times larger (in practice much more) than the number of events in this example. Thus, in Markov Simulation the RNG calls are to check whether an event might occur in the next time-step.

In Poisson Simulation, on the other hand, the aggregated flow equation is used where, typically, $\mathrm{n}$ events will happen during $(t, t+\Delta t)$. A primitive Poisson Random Number Generator then makes $n+1$ calls to a uniform RNG. (For larger $n$, say $n>12$, we can be still more effective.) We thus have about one call to a uniform RNG for each event that really happens. It is these three points combined (aggregation over state-space, aggregation over time and a reduced number of RNG calls for events rather than for possible events) that makes execution of a Poisson Simulation orders of magnitude faster than execution of a Markov model.

(4) Since Poisson Simulation is an extension of Continuous System Simulation (CSS), an important aspect is to preserve the time-scaling property in Poisson Simulation. This is achieved by the $P o[\Delta t \cdot \sim] / \Delta t$ construction used in the flow rate equations. The time scaling is used to find a time-step small enough to handle the dynamics of the model. This means that $\Delta t=\varepsilon$ and $\Delta t=\varepsilon / 2$ should produce sufficiently similar results (from the perspective of the actual purpose). The way to test this is to try different step-sizes on the embedded deterministic model to find out whether $\Delta t$ is small enough. This also works well as a first try for stochastic models, although different values of $\Delta t$ should be tested for the stochastic case comparing resulting pdfs.

For a Markov model a time-scaling mechanism is lacking. It is then problematic to decide what time-step $h$ should be used to comply with the underlying construction: $P\left[E_{i} \rightarrow E_{j}\right.$ in $t, t+h]=a_{i j} h+o(h)$.

(5) The Continuous System Simulation concept on which Poisson Simulation is based gives a rich function library, even including table look-up functions for one or several variables. Thus any empirically found relationship can simply be plugged into the model without any mathematical treatment or complication. It is also a great advantage that it can be directly written and executed in any CSS language with its function library, random number generators, output facilities, etc.

To summarise, the use of state variables in Poisson Simulation instead of a state set in the Markov approach makes the model very much smaller. This aggregation into state variables, the aggregation of events over time and the execution of events that actually happen instead of single events that might happen makes Poisson Simulation orders of magnitude faster than Markov Simulation. Furthermore, much larger and more complicated models can be built and executed with Poisson Simulation than is possible with the Markov approach.

We do not know of any non-trivial case where the simulation of a Markovian population model is smaller, easier, faster or has any other merits than Poisson Simulation in the numerical context. 


\section{References}

[1] M.S. Bartlett, Stochastic Population Models in Ecology and Epidemiology, Wiley, NY, 1960.

[2] R. Lande, S. Engen, B.-E. Saether, Stochastic Population Dynamics in Ecology and Conservation: An Introduction, Oxford University, Oxford, 2003.

[3] J.H. Matis, T.R. Kiffe, Stochastic Population Models: A Compartmental Perspective: v. 145, Lecture Notes in Statistics S. Springer, NY, 2000.

[4] J.R. Clymer. Systems Analysis Using Simulation and Markov Models Prentice Hall International Series in Industrial \& Systems Engineering. NJ, 1991.

[5] C.J. Mode, C.K. Sleeman, Stochastic Processes in Epidemiology: HIV/AIDS, Other Infectious Diseases and Computers, World Scientific Publishing Co., Singapore, 2000.

[6] G.S. Fishman, Principles of Discrete Event Simulation, John Wiley \& Sons, NY, 1978.

[7] J. Banks, J.S. Carson, B. Nelson, D. Nicol, Discrete Event Simulation, Prentice Hall, NJ, 2005.

[8] V. Grimm, S.F. Railsback, Individual-Based Modeling and Ecology (Princeton Series in Theoretical \& Computational Biology), Princeton University, NJ, 2005.

[9] G.A. Korn, J.V. Wait, Digital Continuous-system Simulation, Prentice Hall, NJ, 1978.

[10] F.E. Cellier, E. Kofman, Continuous System Simulation, Springer, NY, 2006.

[11] L. Gustafsson, Poisson Simulation - A Method for Generating Stochastic Variations in Continuous System Simulation, Simulation 74 (5) (2000) 264.

[12] W. Kreutzer, System Simulation: Programming Styles and Languages, Addison-Wesley Publishing Company, Inc., Sydney, 1986.

[13] M.S. Hamilton, Estimating Length and Orders of Delays in System Dynamics Models, in: J. Randers (Ed.), Elements of the System Dynamics Method, The MIT, MA, 1980, p. 162.

[14] W.O. Kermack, A.G. McKendrick, Contributions to the Mathematical Theory of Epidemics, Proc. Royal Soc. A 115 (1927) 700.

[15] N.T.J. Bailey, The Mathematical Theory of Infectious Diseases and its Applications, Griffin, London, 1975.

[16] R.M. Anderson, R.M. May, Infectious Diseases of Humans: Dynamics and Control, Oxford University, Oxford, 1991.

[17] H. Andersson, T. Britton, in: Stochastic Epidemic Models and Their Statistical Analysis, Lecture notes in Statistics, 151, Springer, NY, 2000.

[18] E. Renshaw, Modelling Biological Populations in Space and Time, Cambridge University, Cambridge, NY, 1991.

[19] A.M. Law, W.D. Kelton, Simulation Modeling and Analysis, McGraw-Hill Inc., NY, 1991.

[20] P. Bratley, B.L. Fox, L.E. Schrage, A Guide to Simulation, Springer, NY, 1983.

[21] J.L. Devore, Probability and Statistics for Engineering and Sciences, Sixth edition., Thomson Learning, Inc., Toronto, Canada, 2004.

[22] W.H. Press, B.P. Flannery, S.A. Teukolsky, W.T. Vetterling, Numerical Recipes in Pascal - The Art of Scientific Computing, Cambridge University, Cambridge, UK, 1989, The book is also available in FORTRAN or C.

[23] L. Gustafsson, Studying dynamic and stochastic systems using Poisson Simulation, in: H. Liljenström, U. Svedin (Eds.), Micro-Meso-Macro: Addressing Complex Systems Couplings, World Scientific Publishing Company, Singapore, 2005, p. 131.

[24] L. Gustafsson, Poisson Simulation as an extension of CSS for the modeling of queuing systems, Simulation 79 (9) (2003) 528.

[25] L. Gustafsson, Tools for Statistical Handling of Poisson Simulation: Documentation of StocRes and ParmEst, Dept. of Biometry and Engineering, The Swedish University of Agricultural Sciences, 2004.

[26] T. Hedqvist, Wanda for MATLAB. Methods and Tools for Statistical Handling of Poisson Simulation, Uppsala University, ISSN: 1401-5749, UPTEC IT0422, Uppsala, Sweden, 2004.

[27] Powersim Reference Manual, Powersim Corporation, 1175 Herndon Parkway, suite 600, Herndon, VA 22170, USA, Powersim Press, 1996. (A free demo to test the examples in this paper is found at: www.powersim.com. Note for using the statistical tools the so called Powersim Constructor should be used.).

[28] Using MATLAB, The MathWorks, Inc. Natick, MA., 2002.

[29] Turbo Pascal ${ }^{\circledR}$ User's Guide. Version 6.0. Borland International, CA, 1990. 
[30] M. Greenwood, On the statistical measure of infectiousness, J. Hyg. Camb. 31 (1931) 336.

[31] M. Braun, Differential Equations and Their Applications, Springer, NY, 1993.

[32] J.W. Haefner, Modeling Biological Systems: Principles and Applications, Chapman and Hall, International Thomson Publishing, NY, 1996.

[33] V. Volterra. "Variazioni e fluttuazioni del numero d'individui in specie animali conviventi." Memoire della R. Accademia Nazionale dei Lincei, anno CCCCXXIII, II, 1926, pp. 1-110.

[34] P.E. Waggoner, Progress curves and Foliar diseases: their interpretation and use, in: K.J. Leonard, W.E. Fry (Eds.), Plant Disease Epidemiology - Population Dynamics and Management, 1, Macmillan Publishing Company, NY, 1986.

[35] D. Clayton, M. Hills, Statistical Models in Epidemiology, Oxford University, Oxford, 1993.

[36] F. Elinder, B. Frankenhaeuser, P. Århem, Non-stationary analysis of the Na current in myelinated nerve fibers of Xenopus laevis: experiments and stochastic simulations, BioSystems 62 (2001) 13.

[37] D.G. Luenberger, Introduction to Dynamic Systems: Theory Models and Applications, John Wiley \& Sons Inc., NY, 1979.

[38] F. Lanchester, Aircraft in Warfare, the Dawn of the Fourth Arm. Constable: London, UK, 1916.

[39] L. Ljung. System Identification - Theory for the User. Prentice-Hall Ptr. Upper Saddle River, New Jersey, second ed., 1999.

[40] H. Tong, Non-linear Time Series - A Dynamic System Approach, Oxford University, Oxford, 1990.

[41] D. Cooke, A.H. Craven, G.M. Clarke, Basic Statistical Computing, Edward Arnold Ltd., London, 1982. 